\title{
The Effects of Negation Mode, Syllogistic Invalidity, and Linguistic Medium on the Psychometric Properties of Deductive Reasoning Tests
}

\author{
Mary Ant Mescer and Magd Colberg \\ U.S.OFtice or Prisonmel Maragement
}

This study explored the relationship between certain formal (logical) propertics of syllogistic items and the psychometric properties of the items. The formal properties of negation mode, syllogistic invalidity, and linguistic medium (symbolic versus verbal) were varied in 16 syllogistic tests. When administered to large samples of college-educated adults, the tests had difficulty levels that were in consonance with previous findings relative to the content and validity of syllogisms. However, the variable of negation mode had the greatest effect on test means. The study expands on previous studies of negation by analyzing negative propositional equivalents in the syllogistic context. These equivalents include term negation in addition to the more familiar verb negation.

An analytical review of the history of psychometrics in the deductive area reveals that the formal (logico-mathematical) systems of syllogistic logic, and of deductive logic in general, have not been explored to the fullest extent possible. One of the principal areas of neglect, for example, is that of propositional variables, particularly negative propositional variables. In the area of negation, studies have been largely limited to negative subsumption (i.e., to the use of the negative copula to be, as in No $A$ are $B$ ) and have not dealt with negation in the context of terms, i.e., with contrapositives and inverses of propositions in which conceptual ne-

APPLIED PSYCHOLOGICAL MEASUREMENT

Vol. 8, No. 1, Winter 1984, pp. 71-79

(C) Copyright 1984 Applied Psychological Measurement Inc. 0146-6216/841010071-09\$1.70 gation accompanies subsumptive negation, as in Some non-B are not non-A.

The fundamental purpose of the study presented in this paper was to vary certain formal (logical) properties of syllogistic items in order to explore their influence on the psychometric properties of syllogistic tests. These formal properties were the negation mode (conceptual as well as subsumptive), syllogistic invalidity versus syllogistic validity, and the symbolic versus the verbal medium. The presentation of this study will be preceded by a very brief discussion of negative propositional equivalents and by a summary of the treatment given in most major psychometric studies and in the experimental literature to the logical properties which are relevant to this study.

\section{Negative Rquivalenes}

The propositions which are used in categorical syllogisms (and in other deductive forms) are of four types: Universal Affirmative (All $S$ are $P$ ), represented by the symbol A; Universal Negative (No $S$ are $P$ ), represented by the symbol $\mathbb{E}$; Particular Affirmative (Some $S$ are $P$ ), represented by the symbol I; and Particular Negative (Some $S$ are not $P$ ), represented by the symbol $O$.

These propositions have negative equivalents that can be inferred through processes called obversion, obverted conversion, contraposition, and inversion. These processes, particularly the last two, are based on the contradiction, with respect to the orig- 
inal proposition, of both the copula to be and one or both terms. For example, the converse of the $\mathbb{E}$ proposition is No $P$ are $S$, the obverse of the I proposition is Some $S$ are not $\bar{P}$, the obverted converse of the E proposition is All $P$ are $\bar{S}$, an inverse of the A proposition is Some $\bar{S}$ are $\bar{P}$, and the contrapositive of the $E$ proposition is Some $\bar{P}$ are not $\bar{S}$. (A line over a term denotes negation of the term.) The reader who is interested in exploring the valid inference of negative propositional equivalents is referred to Cohen and Nagel (1934) for a succinct delineation of these inferences.

\section{Psychenometric Studies}

The study of Wilkins (1928), which provided the first extensive set of data on syllogistic reasoning in the U.S.A., represents the first time that the difficulty levels of the symbolic and verbal media were studied in both valid and invalid syllogistic questions. Her findings indicated that syllogistic problem solving was more difficult in the symbolic medium in the context of both syllogistic validity and invalidity. Both Thurstone and Guilford used syllogistic tests in their factor analytic studies of reasoning abilities. Neither of them, however, analyzed the syllogistic variables that are relevant to the present study. Thurstone, in particular, juxtaposed a variety of schemata from the domains of sentence logic (e.g., conditionals) and predicate logic (e.g., caregorical syllogisms) in all of his rests (Thurstone, 1938a, 1938b, 1940). This juxtaposition of schemata from different domains of deductive logic could possibly account for Thurstone's finding of unclear results and multiple factor loadings in the area of deduction. The early studies of Guilford and his associates used several forms of syllogistic tests, including one of Thurstone's logically heterogeneous tests. The unclear results that were obtained caused these researchers in one study to question whether or not deduction constituted a human ability (Guilford, Christensen, Kettner, Green, \& Hertzka, 1954). However, in Guilford's later work, in which he developed the Structure of Intellect model, deduction was found to fit into the mental operation category of "convergent pro- duction," which was defined as the "Generation of logical conclusions from given information, where emphasis is upon achieving unique or conventionally best outcomes. It is likely that the given (cue) information fully determines the outcome, as in mathematics and logic" (Guilford \& Hoepfner, 1971, p. 20). This suggested identity between evidence and conclusion represented a considerable step forward in the evolution of the definition of deduction (and, by implication, of syllogistics) in psychometrics.

In the 1963 kit of reference tests for cognitive factors (French, Ekstrom, \& Price) Syllogistic Reasoning was defined as the ability to reason from stated premises to their necessary conclusions, a definition perfectly consonant with the logicomathematical definition of deduction. Syllogistic Reasoning was identified with Thurstone's Deduction and Guilford's Logical Evaluation (a name given in the Hertzka, Guilford, Christensen, \& Berger, 1954, study to a factor on which many syllogistic tests loaded). In the 1976 kit Syllogistic Reasoning became Logical Reasoning and was defined as "the ability to reason from premise to conclusion or to evaluate the correctness of a conclusion" (Ekstrom, French, \& Harman, 1976, p. 141). This definition is also consonant with the logicomathematical definition of deduction in general and syllogistics in particular, although it is stated in such broad terms that it can be said to be equally applicable to induction (cf. Colberg, Nester, \& Cormier, 1982).

\section{Experimenental Studies}

Among the studies in experimental psychology that are of relevance to the present study, many have discovered that invalid syllogisms are, on the average, more difficult than valid syllogisms (e.g., Dickstein, 1975; Johnson-Laird \& Steedman, 1978; Woodworth \& Sells, 1935). Dickstein (1976) varied the proportion of invalid syllogisms in order to see if response bias contributed to the tendency of subjects not to answer "No valid conclusion." He found that subjects were not influenced to avoid the response "No valid conclusion" by the number 
of invalids contained in the test. The present study will provide a further test of this hypothesis with proportions of invalids that are more realistic for a multiple-choice test.

In regard to the linguistic medium in which syllogistic items are presented, Guyote and Stemberg (1981) have obtained results analogous to those of Wilkins (1928). They presented the same set of 20 syllogisms in three verbal forms: factual, counterfactual, and anomalous. Their subjects performed better on the factual than on the other two types, which had identical percentages of correct responses.

One source of variation which has not been explored by experimentalists is that of using negative responses, such as contrapositives and inverses, which are logically equivalent to the more usual propositions $\mathrm{A}, \mathbb{E}, \mathbb{I}$, and $\mathrm{O}$ and which contain term negation in addition to verb negation. Wason and Johnson-Laird's summary (1972) of the research on negation, which is limited to verb negation and does not include term negation, reveals that subjects consistently made more errors and took more time to evaluate or to construct megative sentences than they took to perform the same operations with affirmative sentences. Some of their subjects reported converting negative sentences to affirmative ones before responding to them. The authors argued that understanding a negation necessitates understanding the implicit preconception that the negation demies, and they warned that the process of understanding may break down when many negatives are used. Although Wason and Johnson-Laird did not study response choices containing negated terms, their findings on verb negation would lead to the expectation of increased difficulty in response choices containing negated terms as well as negated verbs.

\section{Method}

Sixteen 20-item tests were constructed. Eight were constructed using symbols for the three syllogistic terms and eight using verbal language for those terms. The eight verbal tests were constructed to be identical in logical structure to the eight symbolic tests. The two versions were used in order to discern the effect of manipulating symbolic terms in comparison to the effect of manipulating verbal terms.

The logical structure of the basic eight tests was designed as follows. There were four sets of paired tests. Each pair contained identical tests as far as both propositional and syllogistic variables are concerned, but in one test the five alternative answers represented converses of the premises or other propositional manipulations of the premises and conclusion, whereas in its "paired" test the alternative answers were presented in negative form, i.e., in contrapositive, inverse, and obverted-converse form. These negative alternatives represent manipulations (valid or invalid) performed on the same premises as those on which manipulations were performed in the affirmative paired test. For example:

All $v$ is $w$. Some $x$ is not $w$.

Therefore,

A) Some $w$ is $v$. (limited converse of major)

B) Some $x$ is not $v$. (conclusion)

C) All $w$ is $v$. (invalid converse of major)

D) Some $w$ is not $x$. (invalid converse of minor)

E) No $w$ is $x$. (superaltern of invalid converse of minor)

Therefore,

A) Some non- $y$ is not $w$. (inverse of major)

B) Some non- $y$ is not non- $x$. (contrapositive of conclusion)

C) All non-w is non- $v$. (contrapositive of major)

D) Some non- $x$ is $w$. (invalid inverse of minor)

E) Some non-w is not non- $x$. (contrapositive of minor)

The instructions for all tests were identical: The syllogism was explained, and an example was provided. In the negative versions of the tests, a sentence was added stating that conclusions were to be reached abour negative concepts from statements about affirmative concepts. In the symbolic versions of the tests, a sentence was added stating that in those tests letters of the alphabet represented 
the concepts or terms. The time limit for each test was 35 minutes.

Out of the four sets of paired tests, one set was constructed exclusively with valid syllogisms. These included the basic 19 valid forms plus one subaltern syllogism, which was included in order to complete the 20 -item test.

The negative versions of the paired tests constructed only with valid syllogisms contained five inverses, nine contrapositives, and six obverted converses in the correct alternative. This distribution was dictated by the validity of these inferential processes of equivalence for each statement $A, E, \mathbb{I}$, and $\mathrm{O}$. For example, an I conclusion is not susceptible to contraposition or inversion.

The remaining three pairs of tests contained invalid syllogisms: One pair contained nine invalid syllogisms, another pair contained five, and the last pair contained four. The three pairs of tests containing invalid syllogisms included "none of these" as one of the five alternative answers on all questions, whereas the tests containing only valid syllogisms did not include such an alternative. The five invalid syllogisms contained in one pair and the four contained in the other pair were the same as the nine contained in the first pair. The smaller numbers of invalids were included in order to study the performance of the "none of these" answer as a correct choice in approximately one-fifth of the questions. This was considered important because in multiple-choice tests the expectation, and the general rule, is that all response alternatives (A, $B, C, D, E)$ will be correct equally often. Hence, in a multiple-choice test with five response choices per question, the alternative $E$, which has traditionally been the "none of these" answer, would be expected by subjects to be correct on approximately one-fifth of the questions.

The determination to study exactly nine invalid syllogisms (among the large number of such syllogisms) was based on the traditional axiomatic and theorematic foundations of syllogistic logic (cf. Cohen \& Nagel, 1934). That is to say, the invalid syllogisms used were constructed in violation of one of the traditional five axioms or one of the traditional four "general theorems" (applicable to all four figures). Additionally, two invalid syllog- isms were constructed to express a contradiction of one of the "special theorems" (applicable to individual figures).

The 16 tests were administered as an experimental part in a Federal employment test battery. The tests were given to large ( $N$ was approximately 1,500 for each test) nationwide random samples of the people who took the test in April, 1979. Previous studies (Wing, 1980) indicated that approximately $80 \%$ of the applicants for this examination were college seniors or graduates.

\section{虽esulits}

Table 1 presents the means, standard deviations, and KR-20 reliabilities of the 16 experimental tests. Each test was scored on a number-correct basis only; the means reported refer to the number of questions answered correctly. That the time limit was adequate for the test is confirmed by the finding that on 10 of the tests at least $99 \%$ of the applicants answered the last item of the test, on two of the tests $98 \%$ answered the last item, and on four of the tests $97 \%$ answered the last item. All protocols were included in the analyses, whether or not the applicant completed the test, since it was determined that including protocols with unanswered items had a negligible effect on the analysis.

The information reported in Table 1 reveals that the tests with negative response alternatives were more difficult than those with affirmative alternatives, that syllogisms in symbolic form were more difficult than those in verbal form, and that the total score was affected by the number of valid syllogisms in the test. The tests with 20 valid syllogisms were considerably easier than those which had only 11 valids, and the tests with 15 and 16 valids were intermediate in difficulty.

A $2 \times 4 \times 2$ fixed effects analysis of variance was performed on the test scores. The method of unweighted means was used because the $N \mathrm{~s}$ were unequal and disproportional on the 16 tests. The summary of the analysis is presented in Table 2 . All three main effects and all four interactions were significant at the .01 level or lower. With such large sample sizes, however, even trivial findings yield significant test statistics. The $\omega^{2}$ statistic was calculated for each main effect and interaction in 
order to assess the percent of variance due to each effect. These statistics are given in Table 2. The negative-affirmative variable contributed $32 \%$ of the variance. The number-of-valids and symbolicverbal variables accounted for only $5 \%$ and $4 \%$, respectively. The interaction between the negativeaffirmative variable and the number-of-valids variable accounted for only $.7 \%$ of the variance, while the other interactions were even more negligible. Because the 15-valid and 16-valid conditions were perhaps too similar to be analyzed by a fixed-effects model, the analysis of variance was performed a second time using only three levels $(11$, 16 , and 20) for the number-of-valids factor. The 16-valid condition was chosen because of its ${ }^{66} \mathrm{re}-$ alistic" percentage (20\%) of questions for which "none of these"' was the correct answer. The results of this analysis were very similar to those of the first analysis. The principal differences were that the number-of-valids factor accounted for $6 \%$ of the variance, the symbolic-verbal factor accounted for $3 \%$ of the variance, and the interaction of these two factors (the $\mathrm{B} \times \mathrm{C}$ interaction) was not statistically significant and accounted for none of the variance.

The KR-20 reliabilities of the 20 -item tests ( $\mathrm{Ta}-$ ble 1) are sufficient in almost all cases to permit the tests to be used operationally as a component

\begin{tabular}{|c|c|c|c|c|c|c|c|c|}
\hline \multirow{6}{*}{$\begin{array}{l}\text { Test and } \\
\text { Number of } \\
\text { Valids }\end{array}$} & \multicolumn{8}{|c|}{ Table 1} \\
\hline & $=z$ & & $s=2=$ & 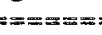 & $s==m=$ & $=-8=5$ & $==$ & $== \pm=$ \\
\hline & \multicolumn{8}{|c|}{ Format of Response Choices } \\
\hline & \multicolumn{4}{|c|}{ Affirmative } & \multicolumn{4}{|c|}{ Negative } \\
\hline & \multirow[b]{2}{*}{$\mathrm{M}$} & \multirow{3}{*}{\multicolumn{2}{|c|}{$\begin{array}{ll} & \mathrm{KR}- \\
\mathrm{SD} & 20\end{array}$}} & \multirow[b]{2}{*}{$\mathbb{N}$} & \multirow[b]{2}{*}{ M } & \multirow{2}{*}{\multicolumn{2}{|c|}{$\begin{array}{ll} & \mathrm{KR}- \\
\mathrm{SD} & 20\end{array}$}} & \multirow[b]{2}{*}{$\mathrm{N}$} \\
\hline & & & & & & & & \\
\hline \multicolumn{7}{|l|}{ Symbolic } & & \\
\hline 20 & 12.78 & 5.26 & .89 & 1,473 & 6.24 & 4.78 & .86 & 1,482 \\
\hline 16 & 10.90 & 4.10 & 80 & 1,537 & 5.32 & 3.73 & .71 & 1,558 \\
\hline 15 & 9.85 & 4.09 & .80 & 1,515 & 5.21 & 3.30 & .78 & 1,520 \\
\hline 11 & 8.71 & 3.70 & .75 & 1,577 & 4.24 & 2.67 & .59 & 1,596 \\
\hline \multicolumn{9}{|l|}{ Verbal } \\
\hline 20 & 15.05 & 4.33 & .87 & 1,492 & 7.47 & 4.51 & .83 & 1,506 \\
\hline 16 & 12.58 & 3.64 & .76 & 1,557 & 7.17 & 3.42 & .75 & 1,617 \\
\hline 15 & 12.69 & 3.51 & .78 & 1,531 & 7.24 & 3.71 & .70 & 1,534 \\
\hline 11 & 10.91 & 3.47 & .73 & 1,625 & 5.51 & 2.84 & .59 & 1,632 \\
\hline
\end{tabular}

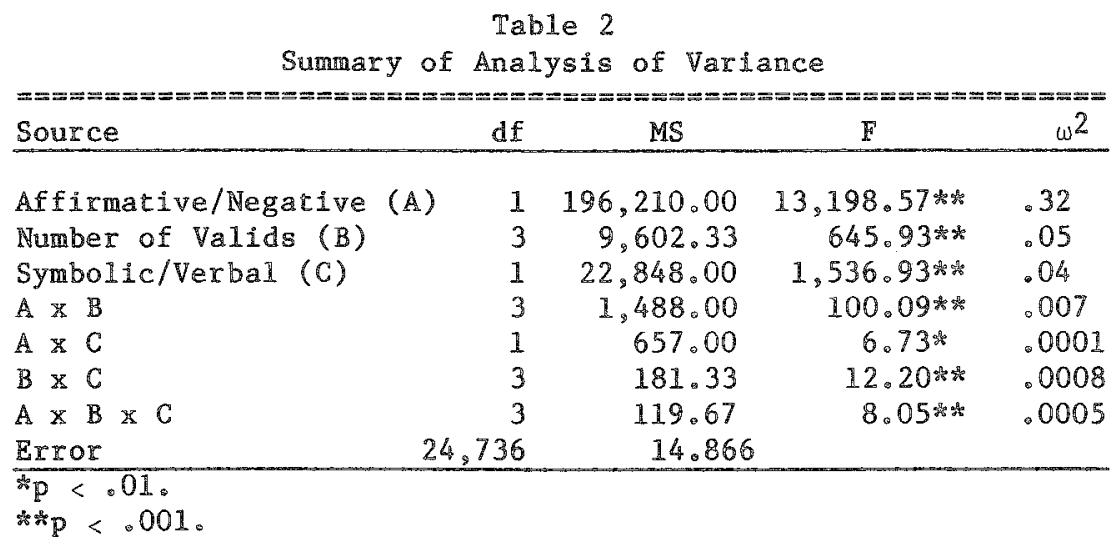

Downloaded from the Digital Conservancy at the University of Minnesota, http://purl.umn.edu/93227. May be reproduced with no cost by students and faculty for academic use. Non-academic reproduction requires payment of royalties through the Copyright Clearance Center, http://www.copyright.com/ 
of the selection test battery. The two tests that have negative response choices and only 11 valid syllogisms are the two exceptions - they both have a KR-20 reliability of .59 . The significance of the effect of the experimental variables on the reliabilities was evaluated through the use of nonparametric tests. Neither the affirmative-negative variable nor the symbolic-verbal variable were shown to have a significant effect on reliability by the Mann-Whitney $U$ test $\left(n_{1}=8, n_{2}=8\right.$ for both significance tests; $p>.05$, in a two-tailed test, for both significance tests). The number-of-valids variable was found to have a significant effect on reliabilities by the Kruskal-Wallis one-way analysis of variance by ranks $(H=10.73, d f=3, p<.02)$. Thus, while the negative-affirmative variable had the greatest effect on test means, the number-ofvalids variable affected test reliabilities.

Table 3 presents the mean proportion correct $(p)$, the mean point-biserial correlation $\left(r_{p b}\right)$, and the mean biserial correlation $\left(r_{b}\right)$ for valid and invalid syllogisms in each of the 16 tests. The pointbiserial and biserial were calculated by correlating the item score with the total score minus item score. In all tests the valid syllogisms were considerably less difficult than the invalids. The difficulty levels of both types of syllogisms were affected analogously by the experimental variables of content (symbols vs. verbal language) and response format (affirmative vs. negative). For example, the valids and invalids both have their highesi average $p$ values in the four tests that have affirmative response choices and that are written in verbal language. They have their lowest average $p$-values on the symbolic test with negative response choices (although the invalids in this case are very close to the invalids in the verbal language-negative test).

The $p$-values of both the invalids and the valids were not affected by the number of invalids in the test. However, the effect of the number of valids is seen in the point-biserial $r s$ and the biserial $r s$ of valid syllogisms. Although the $p$-values of valids tend to remain constant as the number of valids decreases, the point-biserial $r s$ and the biserial $r s$ tend to decrease. This is undoubtedly due to the addition of the very difficult invalid syllogisms to the test. There is no consistent tendency for the point-biserial $r$ s or biserial $r s$ of the invalids to increase as more invalids are added to the test. The point-biserial and biserial rs of the valid syllogisms are much higher than those of the invalids. This is not surprising given the very low $p$-values of the invalid syllogisms. Both the point-biserial $r$ and the biserial $r$ are severely attenuated at low $p$-values due to the effect of guessing (Urry, 1974, 1977). The $r$ s of both types can be seen to increase for the invalid syllogisms as their $p$-values increase. It should also be noted that all of the item-test correlations are positive. (In the entire set of data from which this table is derived, there was only one negative point-biserial $r$, which had a value of -.072 and corresponded to a negative invalid syllogism in the verbal medium.)

Finally, correlations were obtained between scores on valid and invalid syllogisms in the 15-valids tests and in the 11-valids tests. In the 15-valids tests, the scores on the set of five invalid syllogisms were correlated with the scores on a set of five corresponding valid syllogisms. In the 11 -valids tests correlations were found between all 11 valids and the 9 invalids. The correlations are presented in Table 3. The results are similar for the symbolic and verbal items. In the tests with affirmative response choices, the correlations are in the range of .22 to 29 . In the tests with negative choices, the correllations range from .02 to .10 . These latter correlations are low undoubtedly because the iterns were so difficult that a large share of the correct responses was due to guessing. The higher correlations-- those for the affirmative tests-were undoubtedly also attenuated by guessing and by the wide difference in the difficulty levels of the valid and invalid sets of syllogisms (Carroll, 1945). When these correlations were corrected for attenuation due to unreliability (using $\mathbb{K}-20$ estimates of reliability), they ranged from .42 to .54 .

\section{Discussion}

The results of this experiment have demonstrated that negative term variables can be used in the categorical syllogism and that the interaction of these variables with other variables, such as syllogistic validities and invalidities and verbal or

Downloaded from the Digital Conservancy at the University of Minnesota, http://purl.umn.edu/93227. May be reproduced with no cost by students and faculty for academic use. Non-academic reproduction requires payment of royalties through the Copyright Clearance Center, http://www.copyright.com/ 
Table 3

Mean Percent Correct $(\overline{\mathrm{p}})$, Mean Point-Biserial $\mathrm{r}\left(\overline{\mathrm{r}}_{\mathrm{p}}\right)$. and Mean Biserial $x\left(\bar{x}_{b}\right)$ for Valid and Invalid Syllogisms, and Correlation between Valid and Invalid ( $\mathbf{r}_{i v}$ )

\begin{tabular}{|c|c|c|c|c|c|c|c|}
\hline \multirow[b]{2}{*}{ Test } & \multicolumn{3}{|c|}{ Valids } & \multicolumn{3}{|c|}{ Invalids } & \multirow[b]{2}{*}{$r_{i v}$} \\
\hline & $\overrightarrow{\mathrm{p}}$ & $\bar{r}_{\mathrm{pb}}$ & $\overline{\mathrm{r}}_{\mathrm{b}}$ & $\overline{\mathrm{p}}$ & $\bar{r}_{p b}$ & ${\overrightarrow{r_{b}}}_{b}$ & \\
\hline \multicolumn{8}{|c|}{ Symbolic-Affirmative } \\
\hline 20 valids & .639 & .510 & .673 & & & & \\
\hline 16 valids & .625 & .421 & .557 & .225 & .125 & .172 & \\
\hline 15 valids & .573 & .399 & .525 & .252 & .230 & .325 & .29 \\
\hline 11 valids & .604 & .369 & .485 & .229 & .241 & .339 & .28 \\
\hline \multicolumn{8}{|c|}{ Symbols-Negative } \\
\hline 20 valids & .312 & .453 & .599 & & & & \\
\hline $16 \mathrm{valids}$ & .303 & .388 & .515 & .118 & .065 & .105 & \\
\hline 15 valids & .297 & .337 & .453 & .151 & .049 & .082 & .02 \\
\hline 11 valids & .278 & .279 & .378 & .131 & .077 & .129 & .03 \\
\hline \multicolumn{8}{|c|}{ Language-Affirmative } \\
\hline 20 valids & .752 & .466 & .670 & & & & \\
\hline 16 valids & .712 & .371 & .531 & .297 & .210 & .284 & \\
\hline 15 valids & .729 & .368 & .531 & .351 & .191 & .252 & .22 \\
\hline 11 valids & .754 & .340 & .488 & .290 & .253 & .346 & .29 \\
\hline \multicolumn{8}{|c|}{ Language-Negative } \\
\hline 20 valids & .373 & .403 & .528 & & & & \\
\hline 16 valids & .414 & .303 & .394 & .139 & .072 & .111 & \\
\hline 15 valids & .430 & .370 & .476 & .157 & .069 & .110 & .07 \\
\hline $11 \mathrm{valids}$ & .372 & .254 & .330 & .158 & .114 & .175 & .10 \\
\hline
\end{tabular}

symbolic media, can be controlled to produce tests of varying difficulty levels and high reliability.

The variation that had the greatest effect on test difficulty was the use of negative response choices. The percentage correct on tests with negative response choices was approximately one-half as large as the percent correct on tests with affirmative response choices. This finding was in line with the hypotheses of this study and with the work on negation reported by Wason and Johnson-Laird (1972). However, in the present study the variable of negation acquires dimensions that have not been studied before, since the use of contrapositives and inverses as response choices requires the processing of negated terms (often both $S$ and $\mathbb{P}$ ), sometimes in conjunction with a negated copula. The difficulty levels obtained for these negative formulations in the context of invalid syllogisms were so extreme as to exclude them from use in test batteries. On the other hand, many of the negative formulations proved to be accessible to the subjects in the context of valid syllogisms. These results indicate that the two variables (deductive invalidity and negation of terms and copula) can be handled separately but may not be psychometrically appropriate in conjunction, at least for college-level popwlations and below. The implications of this finding uncover a very rich area of research in the field of cognitive processing, i.e., what type of effort is involved in processing as a cognate the disjunction $-(\overline{\mathrm{S}} \overline{\mathrm{P}})$ ? How does this processing effort compare to that involved in processing as a cognate the disjunction - (SP)?

Secondly, it was found that the tests in verbal language were somewhat less difficult than cotresponding tests expressed in symbols. This finding replicates Wilkins' (1928) finding with her more heterogencous deductive tests. The effect of verbal content found in this study was small when compared to the effect of the term negation variable. The differences in p-values of syllogisms produced by the difference in test content was approximately 
.10 on the average. The verbal language and symbolic tests appear to have been affected in the same way by the other experimental variables. This is reflected in the insignificant contribution of interactions to the explained variance in the analysis of variance. This finding might suggest that reasoning in simple verbal language and reasoning in symbols have substantial commonalities. However, since correlations between individual differences in the two modes are not available from the present study, this suggestion should be regarded as tentative.

In consonance with mamy other studies, this study has shown also that reasoning with invalid syllogisms is considerably more difficult than reasoning with valid syllogisms. The finding of Dickstein (1976) that the difficulty of invalid syllogisms is not affected by the proportion of invalid syllogisms in the test was reproduced with a smaller proportion of invalids than Dickstein used. The present study has shown that even with a realistic proportion (20 $1025 \%$ ) of invalid syllogisms in the test, in which there should be no response bias against choosing the "none of these" alternative, invalid syllogisms were as difficult as they were in a test made up of approximately one-half invalid syllogisms. It was found, however, that inclusion of a large proportion of invalid syllogisms reduced the reliability of the tests.

One question that would be relevant to the use of syllogisms in a psychometric context is that of whether or not valid and invalid syllogisms test the same reasoning processes. Dickstein (1975) found a correlation of .43 between performance on valid and invalid syllogisms. In a later study (1976) he found a correlation of .64 for one experimental group, but a correlation of -.002 for another experimental group (the group that solved valid and invalid syllogisms in nearly equal proportions). It should be noted that each of the correlations in the 1976 study was based on data from only 16 subjects. In the present study, with large samples, positive correlations were found between performance on valid and invalid syllogisms when response choices were affirmative. These correlations would no doubt have been higher if the effects of guessing and the difference in difficulty of the two types of syllogisms could have been elimi- nated. Further indications that the invalids may be tapping many of the same processes are apparent in that all of the point-biserial and biserial $r$ s in the tests were positive (with the one exception noted earlier) and that these statistics for invalids tend to increase as the $p$-value increases. An approach to testing this hypothesis would be to construct invalid syllogisms that are more similar in difficulty to the valids included on the tests, aiming for an average $p$-value of .50 or higher. It would then be expected that the point-biserial $r s$ and biserial $r s$ of the invalids would be similar to those of the valids. Even if invalid syllogisms continued under those circumstances to have low item-test correlations, it would seem to be necessary to include them in a test of deductive reasoning, since the finding of "no valid conclusion" is an important part of the domain of deductive reasoning.

Lastly, it has been the intent of the authors to contribute to the elucidation of deductive reasoning as a human function. The deductive domain is a large schematic domain based on both predicate logic and sentence logic. Thus, it should not be surprising to obtain unclear results from factoranalytic studies, such as Thurstone's and Guilford's, based on schematically heterogeneous tests. As indicated by Colberg (in press), the pluralism of deductive schemata lays the foundation for desirable psychometric distinctions between types of deductive reasoning, although all these types fundamentally share the same logico-mathematical definition of inferring mecessary conclusions.

\section{References}

Carroll, J. B . (1945). The effect of difficulty and chance success on correlations between items or between tests. Psychometrika, 10,1-19.

Cohen, M. R., \& Nagel, E. (1934). An introduction to logic and scientific method. New York: Harcourt-Brace.

Colberg, M. (in press). Towards a taxonomy of verbal tests based on logic. Educational and Psychological Measurement.

Colberg, M., Nester, M. A., \& Cormier, S. M. (1982). Inductive reasoning in psychometrics: A philosophical corrective. Intelligence, $6,139-164$.

Dickstein, L. S. (1975). Effects of instructions and premise order on errors in syllogistic reasoning. Jownal of

Downloaded from the Digital Conservancy at the University of Minnesota, http://purl.umn.edu/93227. May be reproduced with no cost by students and faculty for academic use. Non-academic reproduction requires payment of royalties through the Copyright Clearance Center, http://www.copyright.com/ 
Experimental Psychology: Human Learning and Memory, 1, 376-384.

Dickstein, L. S. (1976). Differential difficulty of categorical syllogisms. Bulletin of the Psychonomic Society, $8,330-332$.

Ekstrom, R. B., French, J. W. \& Harman, H. H. (1976). Manual for kit of factor-jefenced cognitive tests. Princeton NJ: Educational Testing Service.

French, J. W., Ekstrom, R. B., \& Price, L. A. (1963). Manual for kit of reference tests for cognitive factors. Princeton NJ: Educational Testing Service.

Guilford, J. P., Christensen, P. R., Kettner, N. W., Green, R. F., \& Hertzk, A. F. (1954). A factoranalytic sudy of Navy reasoning tests with the Air Force Aircrew Classification Battery. Educational and Psychological Measurement, 14, 301-325.

Guilford, J. P., \& Hoepfner, R. (1971). Analysis of intelligence. New York: McGraw-Hill.

Guyote, M. J., \& Sternberg, R. J. (1981). A transitivechain theory of syllogistic reasoning. Cognitive Psychology, 13, 461-525.

Hertzka, A. F., Guilford, J. P., Christensen, P. R., \& Berger, R. M. (1954). A factor-analytic study of evaluative abilities. Educational and Psychological Measurement, 14, 581--597.

Johnson-Laird, P. N., \& Steedman, M. (1978). The psychology of syllogisms. Cognitive Psychology, 10, 64-99.

Thurstone, L. L. (1938a). The perceptual factor. Psychometrika, 3, $1-17$.

Thurstone, L. L. (1938b). Primary mental abilities. Psy- chometric Monographs, No. 1.

Thurstone, L. L. (1940). Experimental study of simple structure. Psychometrika, 5, 153-168.

Urry, V.W. (1974). Approximations to item parameters of mental test models and their uses. Educational and Psychological Measurement, 34, 253-269.

Urry, V.W. (1977). Tailored testing: A successful application of latent trait theory. Joumal of Educational Measurement, 14, 181-196.

Wason, P. C., \& Johnson-Laird, P. N. (1972). Psychology of reasoning: Structure and content. Cambridge MA: Harvard University Press.

Wilkins, M. C. (1928). The effect of changed material on ability to do formal syllogistic reasoning. Archives of Psychology, 16, No. 102.

Wing, H. (1980). Practice effects in traditional mental test items. Applied Psychological Measurement, 4, $141-155$.

Woodworth, R. S., \& Sells, S. B. (1935). An atmosphere effect in formal syllogistic reasoning. Jourral of Experimental Psychology, 18, 451-460.

\section{Anthor"s Address}

Send requests for reprints or further information to Mary Ame Nester, Office of Personnel Research and Development, U.S. Office of Personnel Management, 1900 E. Street, N.W., Room 3G29, Washington DC 20415 , U.S.A.

Downloaded from the Digital Conservancy at the University of Minnesota, http://purl.umn.edu/93227.

May be reproduced with no cost by students and faculty for academic use. Non-academic reproduction requires payment of royalties through the Copyright Clearance Center, http://www.copyright.com/ 